\title{
Durability of partial saturation to counteract liquefaction
}

1 Abdulhakim Zeybek MSc

PhD Candidate, Department of Engineering, University of Cambridge, Cambridge, UK (corresponding author: az323@cam.ac.uk)
2 Gopal Santana Phani Madabhushi MTech, PhD (Cantab) Professor of Civil Engineering, Department of Engineering, University of Cambridge, Cambridge, UK
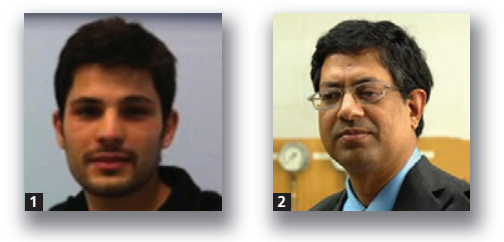

Recently, artificially introducing gas/air into liquefiable soils has been presented as a method for reducing the risks from liquefaction. Although this method offers a simple and cheap solution, its use in practical applications is still very limited. This might be primarily ascribed to the concerns of practising engineers about the durability of gas/air bubbles in soils over time. This paper discusses the durability of entrapped air bubbles under various simulated field conditions that may potentially cause the dissolution, diffusion, compression and escape of air bubbles. Multiple series of $1 \mathrm{~g}$ vertical sand column and high-g centrifuge tests were undertaken to provide insights into the problem. Air-induced partially saturated soils were prepared using an air-injection technique. The test results showed that the majority of entrapped air bubbles in soils can persist under several simulated field conditions for a sufficient period of time, indicating the long-term reliability of the mitigation accomplished.

\begin{tabular}{ll}
\multicolumn{2}{l}{ Notation } \\
$D_{\mathrm{r}}$ & relative density \\
$d_{10}$ & grain diameter at $10 \%$ passing \\
$d_{50}$ & grain diameter at $50 \%$ passing \\
$e_{\max }$ & maximum void ratio \\
$e_{\min }$ & minimum void ratio \\
$G_{\mathrm{s}}$ & specific gravity \\
$\boldsymbol{g}$ & acceleration due to gravity \\
$i$ & hydraulic gradient \\
$k$ & permeability \\
$N$ & nominal centrifugal acceleration \\
$q$ & foundation bearing pressure \\
$S_{\mathrm{r}}$ & degree of saturation \\
$U_{\mathrm{c}}$ & uniformity coefficient
\end{tabular}

\section{Introduction}

Seismic liquefaction of saturated soils is of major concern worldwide since it may cause significant damage to structures. During shaking, the sudden increase in the pore water pressure may lead the soils to lose their shearing resistance. The presence of gas bubbles in saturated soils, and therefore the degree of saturation, $S_{\mathrm{r}}$, is an important factor in geotechnical earthquake engineering since it affects the compressibility of the pore fluid that fills the pores between soil grains. This in turn influences the liquefaction resistance and permeability, $k$, of soils. Laboratory test results have indicated that a small reduction in $S_{\mathrm{r}}$ significantly increases the resistance of soils to liquefaction (e.g. Chaney, 1978). The compressibility of the saturated medium increases with the presence of gas/air bubbles that reduce the generation of excess pore pressure by reduction of volume (Okamura and Soga, 2006).

Soil deposits below the groundwater table are usually considered as fully or nearly fully saturated. Saturated soil deposits, particularly sandy and loose deposits, present a high potential for liquefaction. Considering the positive impact of $S_{\mathrm{r}}$ on liquefaction resistance, a few studies have recently been conducted on the feasibility of artificially introducing gas/air into saturated soils to reduce the liquefaction risk. This has led to the development of a few practical induced partial saturation' techniques that include air injection (Okamura et al., 2006), water electrolysis (Yegian et al., 2007), drainagerecharge and chemical treatment (Eseller-Bayat et al., 2013) and biogas (He et al., 2013).

Execution of the air-injection technique in the field was studied by Okamura et al. (2011). The in situ test results revealed the practicality associated with the use of air injection in reducing $S_{\mathrm{r}}$. Since the only material used in this method was air, it was regarded as a cost-effective and eco-friendly technique (Okamura and Tomida, 2015). The authors conducted experimental research on liquefiable soil deposits to evaluate the feasibility of air injection to mitigate liquefaction beneath shallow foundations. The results demonstrated that liquefaction-induced deformations and associated foundation settlements reduce significantly with a small reduction in $S_{\mathrm{r}}$ (e.g. Zeybek and Madabhushi, 2017a). 


\section{Offprint provided courtesy of www.icevirtuallibrary.com Author copy for personal use, not for distribution}

Although the aforementioned techniques hold promise for use as a liquefaction countermeasure, their use in practical applications is not yet prevalent. This can be basically attributed to the concerns of practising engineers about their reliability on a long-term basis. It is known that the location of a groundwater table in the field is subjected to variations according to the climate and region. The seasonal change of the water table may increase the risk of gas/air bubbles in soils losing their function over time. The gas/air bubbles may escape from the soils and gradually dissolve in groundwater or diffuse under various field conditions, eventually causing an increase in $S_{\mathrm{r}}$ and a reduction in the liquefaction resistance of soil deposits. The durability of undissolved air bubbles introduced during the application of sand compaction piles was investigated by Okamura et al. (2006), showing that entrapped air bubbles in in situ soil deposits survived for 26 years. An experimental investigation of the durability of partial saturation under hydrostatic conditions demonstrated that the $S_{\mathrm{r}}$ of specimens increased by only 2\% in 115 weeks (Eseller-Bayat et al., 2013). The influence of the solubility of oxygen gases in water on $S_{\mathrm{r}}$ was examined as a function of pressure (Gokyer, 2009), demonstrating that the change in $S_{\mathrm{r}}$ was insignificant. The previous research indicated that gas/air bubbles might be persistent, but the combined effects of various field conditions were not accounted for. Very little research is available on the investigation of the potential influences of air durability on the seismic performance of partially saturated soils. Moreover, no such studies have been undertaken to date using centrifuge testing. Therefore, more research is required.

The aim of this study was to investigate the durability of air bubbles under a range of possible field scenarios. Multiple series of $1 \boldsymbol{g}$ sand column and high- $\boldsymbol{g}$ centrifuge experiments were conducted. It is hoped that with the insights offered in this paper these techniques will be widely applied in real engineering projects.

\section{Simplifying hypotheses}

The study presented in this paper is restricted to partially saturated soils with high $S_{\mathrm{r}}$ (usually above $80 \%$ ). Moreover, it is solely concerned with soils with low fines content (e.g. clean sands). The air phase in the soil layers can be in continuous or occluded form, depending on the $S_{\mathrm{r}}$. For partially saturated soils with high $S_{\mathrm{v}}$, air usually takes the form of occluded bubbles and the pore fluid becomes significantly compressible. The diameters of the bubbles are generally smaller than or the same size as the soil particles. Under these conditions, pore air and water are expected to have roughly equal pressure since the surface tension between air and water is not taken into account (Fredlund and Rahardjo, 1993). Figure 1 presents a typical example of the soil conditions before and after air injection and a schematic illustration of the state of the air bubbles in soil. The size of the air bubbles was usually smaller than the size of the soil particles. They were in equilibrium with the surrounding pore fluid. It was assumed that the occluded air

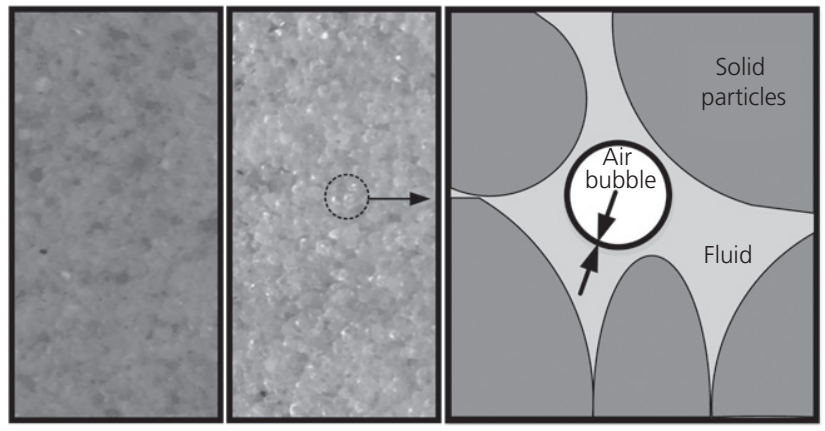

(a)

(b)

(c)

Figure 1. Soil condition (a) before air injection, (b) after air injection and (c) state of air bubbles

bubbles did not significantly interact with the soil structure, and the effect of matric suction was negligible.

In geotechnical centrifuge testing, small-scale models are subjected to an increased gravitational field that is $N$ times the gravitational acceleration $(\boldsymbol{g})$. The scaling laws were derived for 'diffusion or static' events such as consolidation and 'dynamic' events such as earthquakes. The time in diffusion events scales as $1 / N^{2}$ between the model and prototype, whereas it scales as $1 / N$ in dynamic events (Madabhushi, 2014). The durability of entrapped air bubbles in soil layers is known to depend on several factors such as the solubility of air, buoyant forces on bubbles, gravitational acceleration ( $g$-level), water flow, air and fluid pressure, degree of saturation and permeability of soils. Ideally, the derivation of the time-scaling law for the longevity of air bubbles should include the combined effects of these parameters. However, this is difficult due to the complex nature of this analysis. For simplicity, the aforementioned time-scaling factors were considered in this study.

\section{Testing details}

\subsection{Experimental techniques}

An experimental programme consisting of $1 \boldsymbol{g}$ vertical sand column and high-g centrifuge tests was undertaken for the purpose of this study.

For the preparation of soil specimens, a novel one-dimensional (1D) vertical sand column apparatus was designed, and soil specimens were prepared in it (Figure 2). A $1200 \mathrm{~mm}$ clear acrylic tube with an inner diameter of $200 \mathrm{~mm}$ was fixed to a rigid column. Two fittings connected to an overhead tank were available for the saturation of models. Porous plastic was positioned at the bottom to maintain a uniform distribution of infiltrated water. A rubber air curtain hose with several tiny openings of about $0.5 \mathrm{~mm}$ diameter and $5 \mathrm{~mm}$ spacing was connected to the system for air injection. One fitting at the top was connected to a $1 \mathrm{D}$ flow muffler used during the flow tests. 


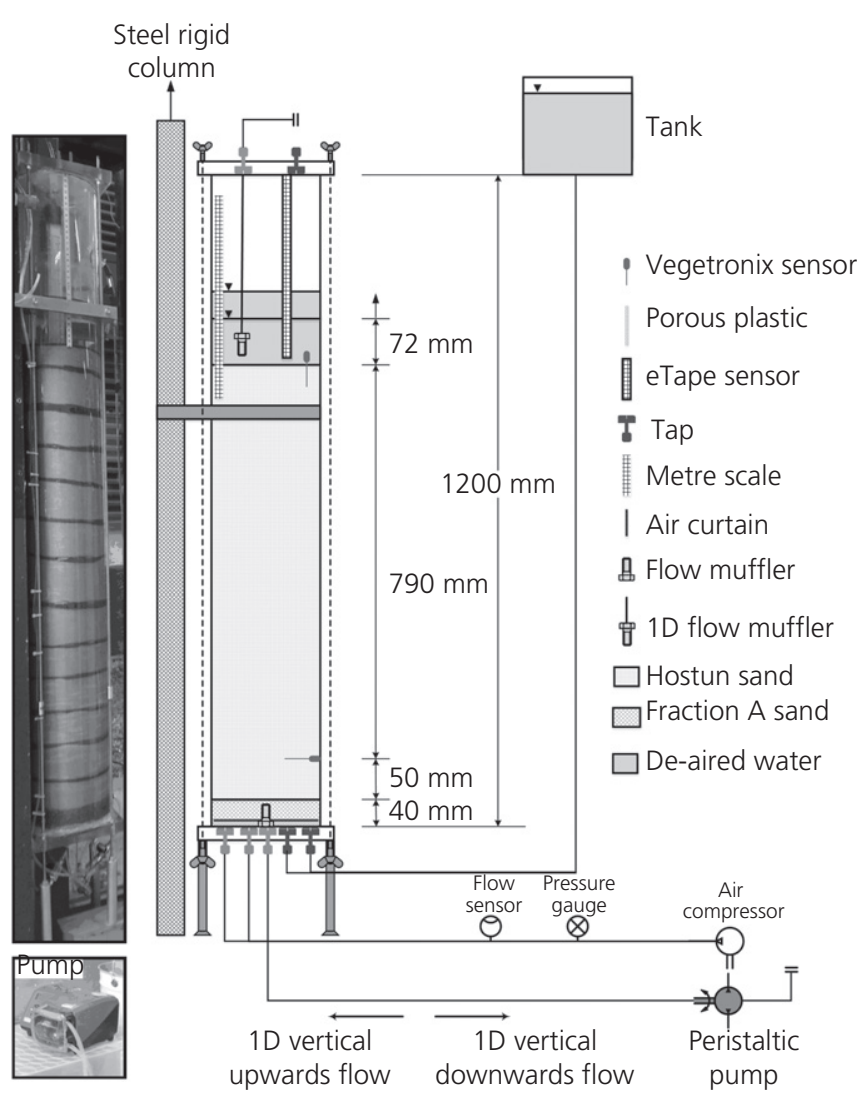

Figure 2. Schematic illustration of the sand column apparatus and typical test set-up

A liquid-level sensor (eTape) was attached to achieve continuous monitoring of the water level, along with a metre scale. A $40 \mathrm{~mm}$ thick drainage layer of fraction A sand was dry pluviated into the tube to obtain 1D flow. Two soil moisture sensors, VH400 (supplied by Vegetronix), were installed at different depths. Dry-pluviated Hostun sand with a relative density, $D_{\mathrm{r}}$, of $40 \%$ was situated on the fraction A sand. The specifications of the Hostun HN31 sand were $d_{10}=0.315 \mathrm{~mm}$, $d_{50}=0.480 \mathrm{~mm}, \quad U_{\mathrm{c}}=1.67, \quad e_{\min }=0.555, \quad e_{\max }=1.01 \quad$ and $G_{\mathrm{s}}=2 \cdot 65$. Uniformly prepared dry specimens were saturated through the infiltration of de-aired water from the bottom up. Subsequently, air was injected into the saturated specimens in a controlled fashion. A peristaltic pump (Watson-Marlow 520S IP31) was used for the flow tests.

A series of centrifuge experiments was conducted on the Turner beam centrifuge at the Schofield Centre in Cambridge University. Homogenous Hostun sand beds were dry pluviated in a rigid container with a Perspex window or laminar box to attain samples at a relative density of $40 \%$. Prepared centrifuge models were spun at different nominal centrifugal accelerations, $N$. The depth of the specimens was $240 \mathrm{~mm}$ in model scale, simulating 16.8 and $9.6 \mathrm{~m}$ soil deposits in prototype scale at $70 \mathrm{~g}$ and $40 \mathrm{~g}$ centrifuge tests, respectively. Arrays of miniature pore pressure transducers and piezoelectric accelerometers were placed at the desired locations. The dry-sand models were saturated with an aqueous solutions of hydroxypropyl methylcellulose using the CAM-Sat system (Madabhushi, 2014). The viscosity of the pore fluid was $N$ times that of water to overcome the incompatibility between the scaling of diffusion and dynamic time. To prepare the partially saturated samples, pressurised air was injected into the saturated soils through the air bubble curtain located on the centre bottom of the model. Lateral excitations were applied to the models using a stored angular momentum actuator device (Madabhushi et al., 1998). Digital images were obtained throughout the tests and particle image velocimetry analysis was performed on the images to obtain the soil displacements (White et al., 2003). The model layouts in prototype scale and a typical set-up of the centrifuge models are presented in Figure 3.

\subsection{Testing programme}

In the field, entrapped air bubbles can be exposed to several field conditions that can potentially cause their dissolution, diffusion, compression or escape. The field conditions were intuitively determined. The possible effects of the simplified field conditions on the durability of air bubbles were simulated and experimentally studied in the laboratory. A summary of the testing programme and typical results from the tests is given in Table 1.

\subsection{Assessment of degree of saturation}

The degrees of saturation of specimens were determined based on the mass-volume relationships and soil moisture sensors (Vegetronix). In the centrifuge tests, the computed $S_{\mathrm{r}}$ values of saturated soils were consistently above $99 \%$, whereas they were $\sim 96 \%$ for the sand column specimens.

Figure 4 illustrates a typical example of the assessment of $S_{\mathrm{r}}$ during the saturation and air-injection process in SCT1. It is obvious that the $S_{\mathrm{r}}$ of the saturated specimen was around $96 \%$. As air was injected, the free-water level increased due to the volume of pore fluid replaced by the volume of air bubbles that entered into the soil. After air injection was halted, the water level dropped to a residual value due to the excess/free air that escaped from the voids of soil. The volume of residual air bubbles in occluded form was considered when calculating the final $S_{\mathrm{r}}$ of partially saturated soils. It is noted that similar final $S_{\mathrm{r}}$ values were achieved from both approaches, but the pattern of the variation of $S_{\mathrm{r}}$ with time differed. This was attributed to the fact that entrapment of air bubbles started from the bottom, leading to earlier variation in the readings of the moisture sensor.

In centrifuge tests, mass-volume relationships and two moisture sensors placed at the mid-depth of the specimens were used to quantify $S_{\mathrm{r}}$. For the first approach, the change in the free-fluid level, and therefore pressure was captured by the pore pressure transducers. The variation of fluid level was also 

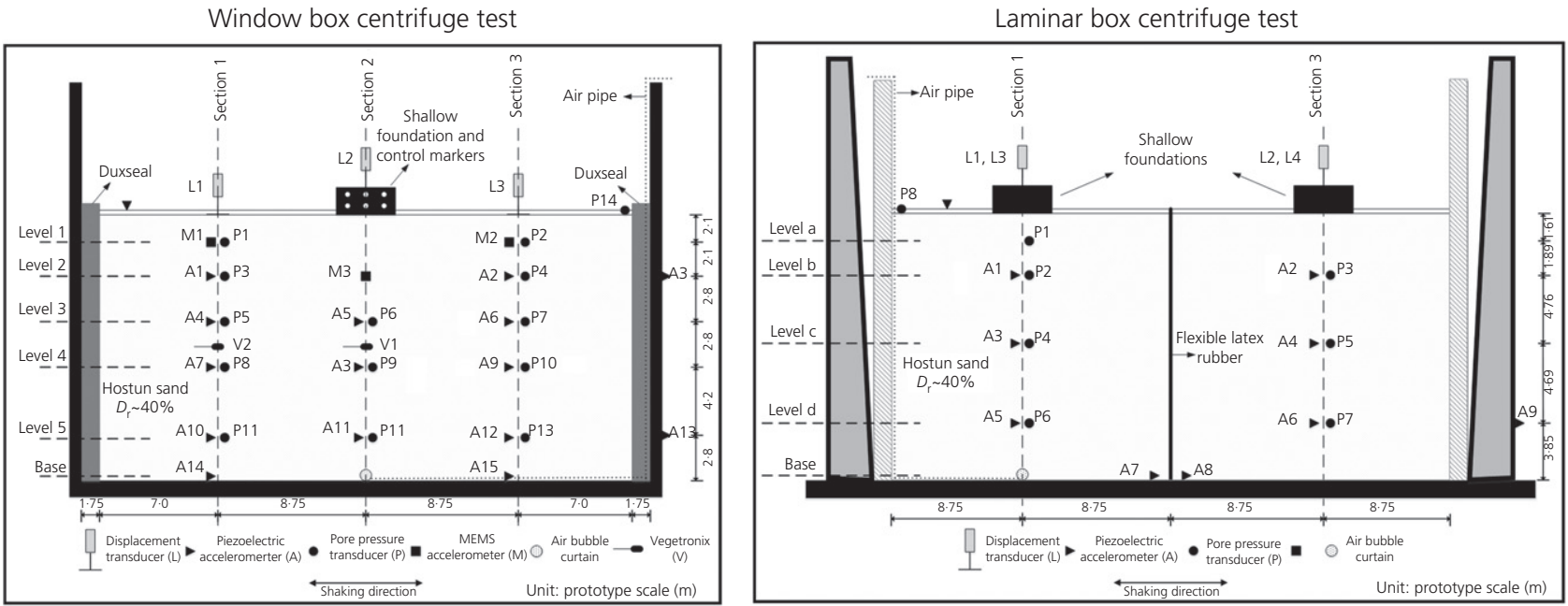

(a)
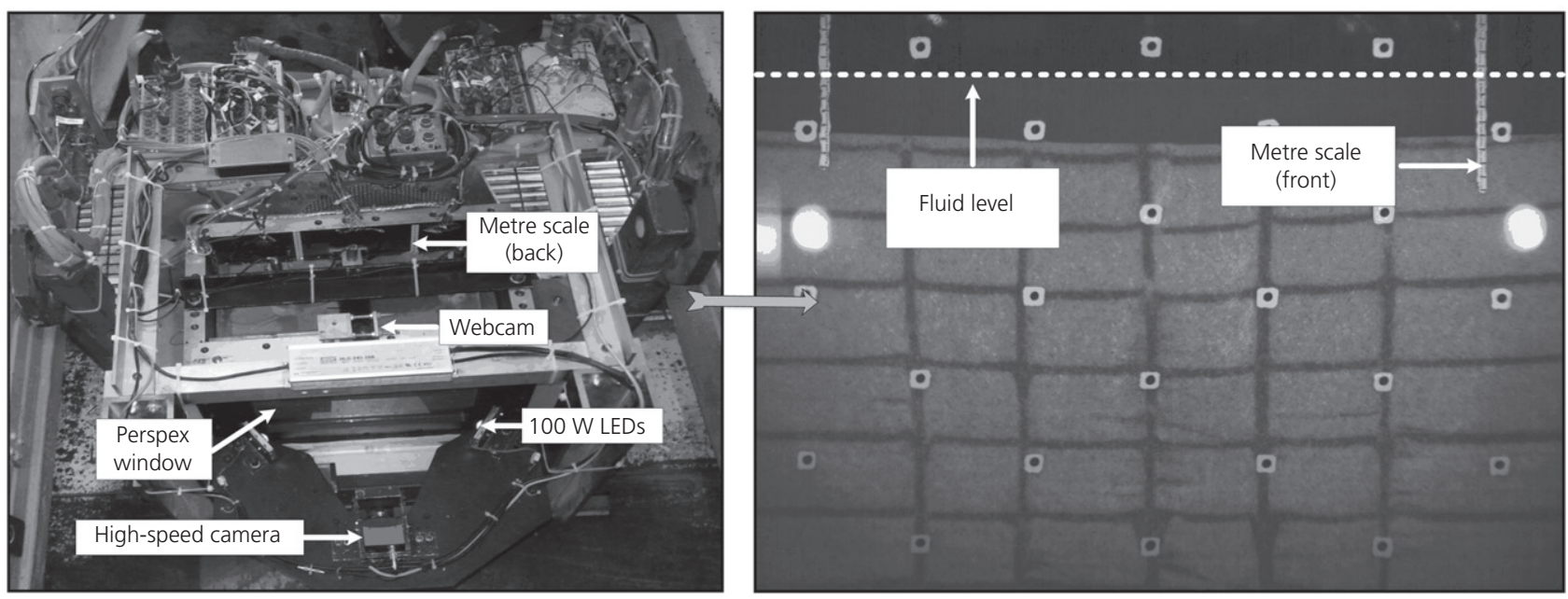

(b)

Figure 3. Cross-section of the centrifuge models and typical test set-up

monitored using the metre scale on the Perspex window (Figure 3).

\section{Analysis and discussion of test results}

\subsection{Durability under hydrostatic conditions}

The durability of entrapped air under hydrostatic conditions (stagnant fluid) was explored at $1 \mathrm{~g}$. Figure 5 presents the change in $S_{\mathrm{r}}$ of partially saturated soils over the short and long term. It can be seen that, in the short term, the increase in average $S_{\mathrm{r}}$ was $\sim 1.4$ and $2 \%$ for sand column tests SCT1 and $\mathrm{SCT} 2$, respectively. The final $S_{\mathrm{r}}$ values recorded by the moisture sensor (bottom) were slightly smaller than those of massvolume relationships. This might indicate that the majority of entrapped air bubbles escaped from the shallow depth of the specimens, and the change in $S_{\mathrm{r}}$ was much greater at the shallow soil layers. It was also observed that the average $S_{\mathrm{r}}$ of specimens increased by $2 \cdot 1$ and $2 \cdot 55 \%$ in 100 days for SCT 1 and SCT2, respectively.

The findings in Figure 5 indicate that the majority of air bubbles can remain entrapped in the voids of specimens under hydrostatic conditions. However, the $1 \boldsymbol{g}$ sand column tests involved only about a $1 \mathrm{~m}$ column of water. In a deep layer of in situ soil, the water pressure will be significantly higher, and such pressure might potentially affect the longevity of air bubbles in soils. High-g geotechnical centrifuge offers an opportunity to recreate the high water pressures in a smallscale model. To evaluate the durability of entrapped air bubbles under larger fluid pressure, the $S_{\mathrm{r}}$ of partially saturated soils was monitored at a centrifugal acceleration of $70 \mathrm{~g}$ over a period of $20-25 \mathrm{~min}$ in model scale (68-85 $\mathrm{d}$ in prototype scale). The larger pore fluid pressures and larger buoyancy forces acting on the air bubbles were the corollary of $16.8 \mathrm{~m}$ 


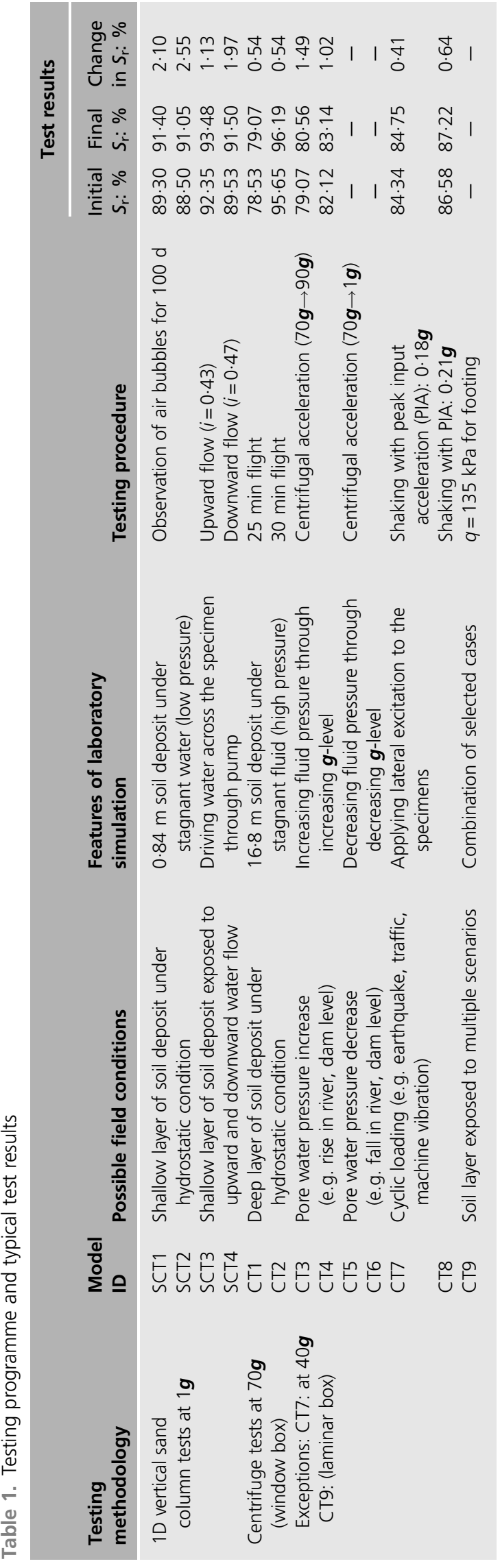

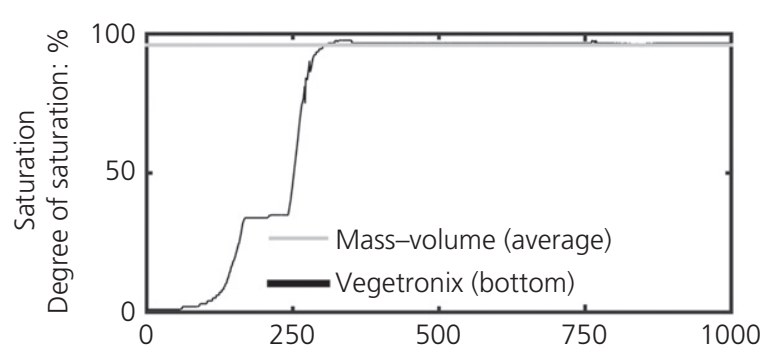
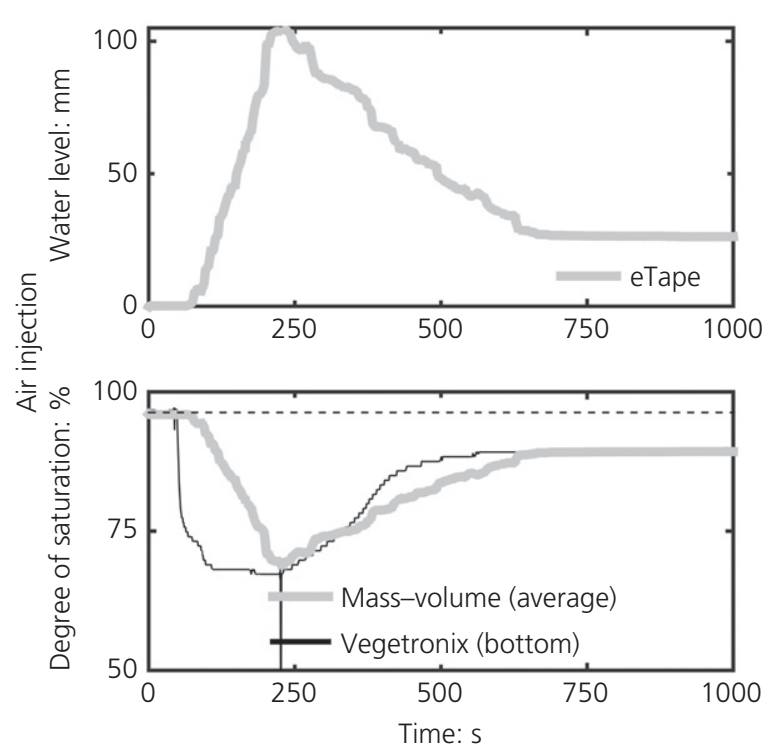

Figure 4. Typical assessment of the degree of saturation of soils (sand column test SCT1)

deep soil deposits in prototype scale. Figure 6 shows the change in $S_{\mathrm{r}}$ under hydrostatic conditions in centrifuge tests CT1 and CT2. It is obvious that the change in $S_{\mathrm{r}}$ was only about $0 \cdot 5 \%$. Since even at larger pore fluid pressures it was very difficult for air bubbles to find a path and escape through a deep soil layer, the change in $S_{\mathrm{r}}$ was mainly attributed to the escape of air bubbles from the shallow soil layers.

\subsection{Durability under hydraulic flow}

The durability of entrapped air bubbles under 1D upward and downward vertical flow was investigated in sand column tests SCT3 and SCT4, respectively. In the tests, water was driven through the partially saturated specimens using a peristaltic pump, with flow continuing for $\sim 30 \mathrm{~h}$. The approximate hydraulic gradients, $i$, applied during the tests were 0.43 and $0 \cdot 47$ for SCT3 and SCT4, respectively.

Figure 7 shows the change of $S_{\mathrm{r}}$ with time during the upward and downward flow. It is evident that the $S_{\mathrm{r}}$ of specimens increased by about $1 \cdot 1$ and $2 \%$ in SCT3 and SCT4, respectively. The change in $S_{\mathrm{r}}$ took place mostly in the first few hours and remained almost unchanged afterwards. This reveals that the volume of occluded air bubbles being carried along by a flow of water through the soil matrix was very small, and the 

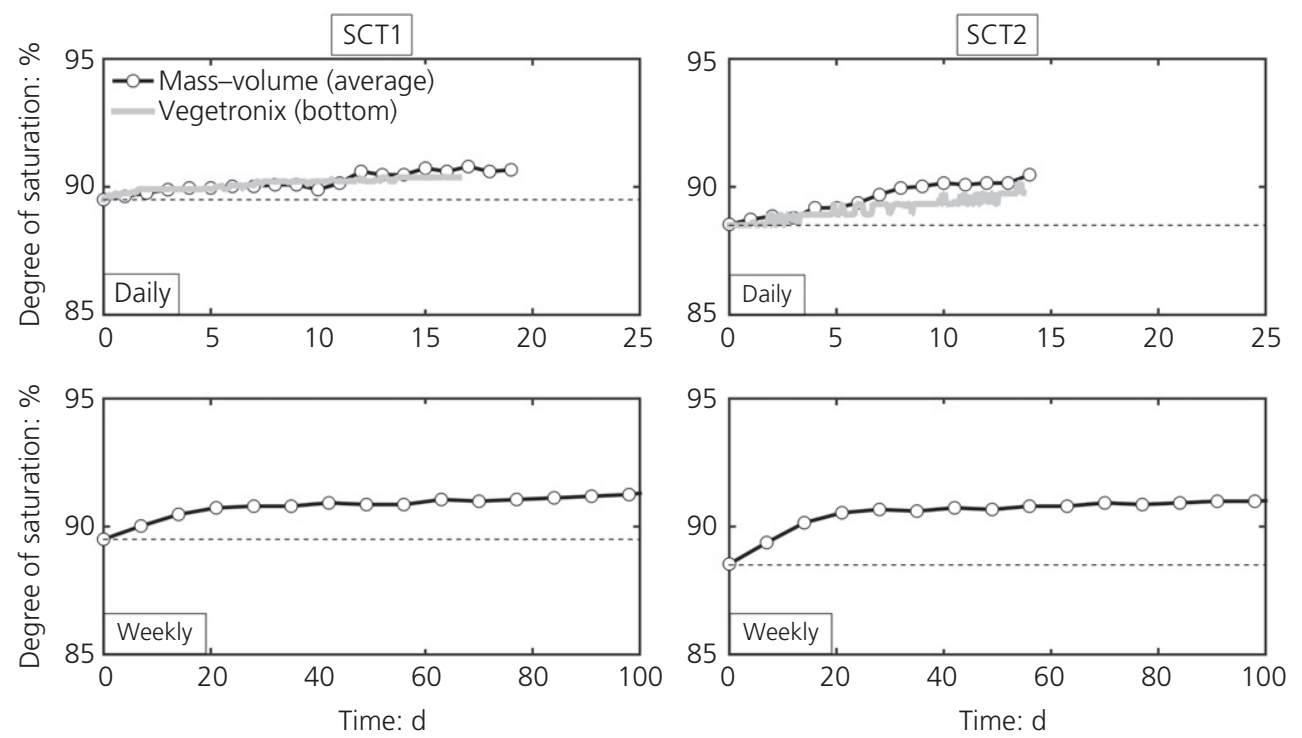

Figure 5. The variation of degree of saturation under hydrostatic conditions at $1 \boldsymbol{g}$ on a short- and long-term basis
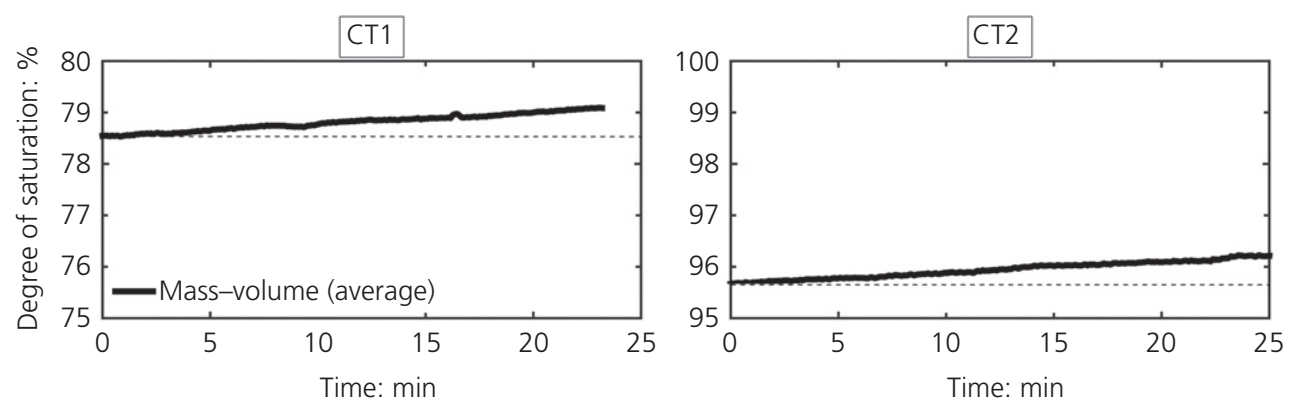

Figure 6. The variation of degree of saturation under hydrostatic conditions at $70 \boldsymbol{g}$ (model scale)
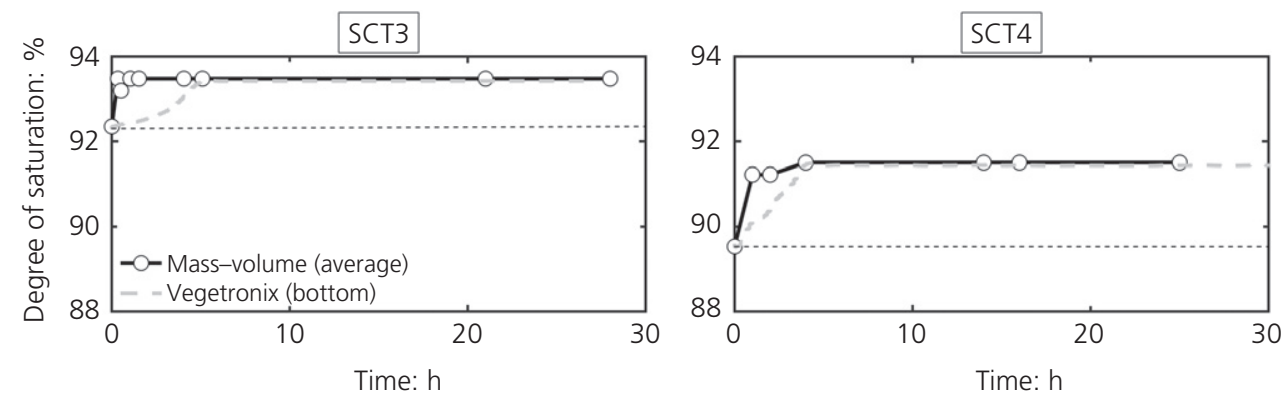

Figure 7. The variation of degree of saturation under upward (SCT3) and downward water flow (SCT4)

majority of air bubbles successfully remained entrapped in the voids of soils. In fact, these results seem to be in accordance with the existing literature (e.g. Eseller-Bayat et al., 2013). It is well known that in saturated soils water flows through the pore space filled with water. However, the permeability of partially saturated soils is lower due to the presence of air bubbles that form a barrier to the water flow. Therefore, instead of travelling with water, the majority of air bubbles are expected to act as a blockage along the flow path.

\subsection{Durability under varying pressure}

The durability of entrapped air in the field can be affected by the pressure change that might be potentially induced by fluctuation of the water level. As stated in Section 2, entrapped 
air bubbles were in equilibrium with the surrounding pore fluid. Intuitively, it may be expected that the changing $\boldsymbol{g}$-level, and therefore the pore fluid pressure would alter the equilibrium condition. Eventually, it would change the volume of entrapped air bubbles, and therefore the final $S_{\mathrm{r}}$ of the specimens. To investigate this hypothesis, a series of four centrifuge tests was conducted.

The change in $S_{\mathrm{r}}$ as a function of $\boldsymbol{g}$-level-time history is shown in Figure 8. The centrifugal acceleration was increased from $70 \mathrm{~g}$ to $90 \mathrm{~g}$ in centrifuge tests $\mathrm{CT} 3$ and $\mathrm{CT} 4$, respectively. This increase refers to a $4.8 \mathrm{~m}$ change in the pore fluid depth. During this process, the change in pore fluid pressures enhanced by centrifugal acceleration and the change in the level of the ground surface and phreatic surface were monitored. The final $S_{\mathrm{r}}$ of specimens was re-calculated based on the final volume of free fluid accumulated above the ground surface. The surface of the pore fluid was also monitored through a webcam to observe potential air bubbles that might escape from the soil due to increased buoyancy forces on them. It is evident that within a $16.8 \mathrm{~m}$ saturated soil profile an increase of $4.8 \mathrm{~m}$ pore fluid pressure caused only 1.49 and $1.02 \%$ increase of $S_{\mathrm{r}}$ in CT3 and CT4, respectively.

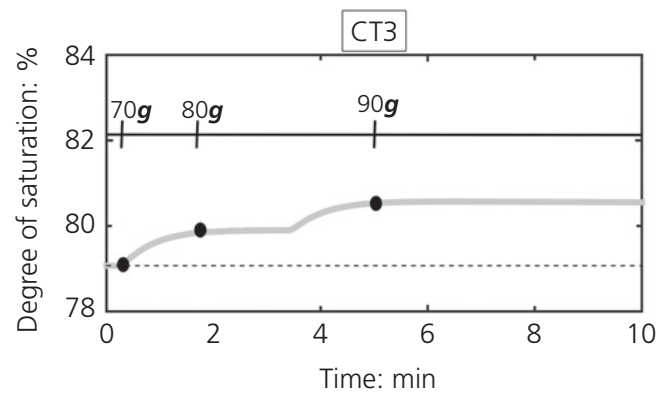

The durability of the air bubbles under decreasing $\boldsymbol{g}$-level and therefore the pore fluid pressure was also investigated in centrifuge tests CT5 and CT6. The centrifugal acceleration was gradually reduced from $70 \mathrm{~g}$ to $1 \mathrm{~g}$. A series of images was taken during this process. Figure 9 shows the images taken at $70 \mathrm{~g}$ and $1 \mathrm{~g}$. The upper images show the condition at which air and pore fluid pressures were in equilibrium at $70 \mathrm{~g}$. Once the centrifugal acceleration reduced to $1 g$, the soil deposits, particularly the shallow layer, started to heave. Some large air-filled cavities became apparent at the mid-depth of soil layers (see the lower images in Figure 9). It was observed that entrapped air bubbles in equilibrium began to move upwards and escaped from the soil surface as the $\boldsymbol{g}$-level was reduced to $\sim 10 \mathrm{~g}$. Due to the decrease in the surrounding pore fluid pressure, the volume of air bubbles increased to reach equilibrium. Air bubbles growing in size tended to coagulate, forming larger bubbles. The formation of air-filled cavities was a direct consequence of the coagulation of relatively smaller air bubbles. The importance of the findings presented here is that any change in the pore fluid pressure affected the air bubble stability at the corresponding soil layers, and led to the escape of air bubbles. In addition, it directly affected the soil behaviour. Significant volume change and softening of soil occurred,

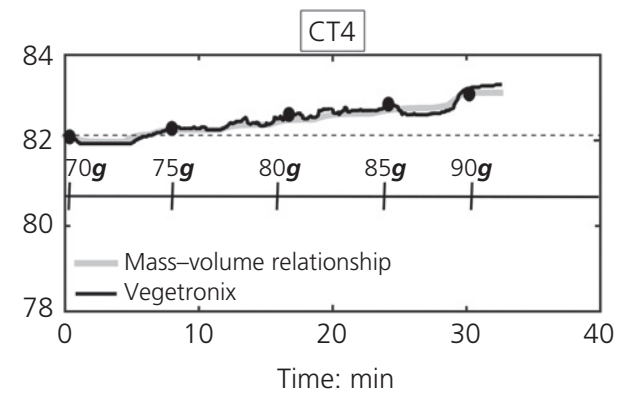

Figure 8. The variation of degree of saturation under increasing $\boldsymbol{g}$-level (model scale)

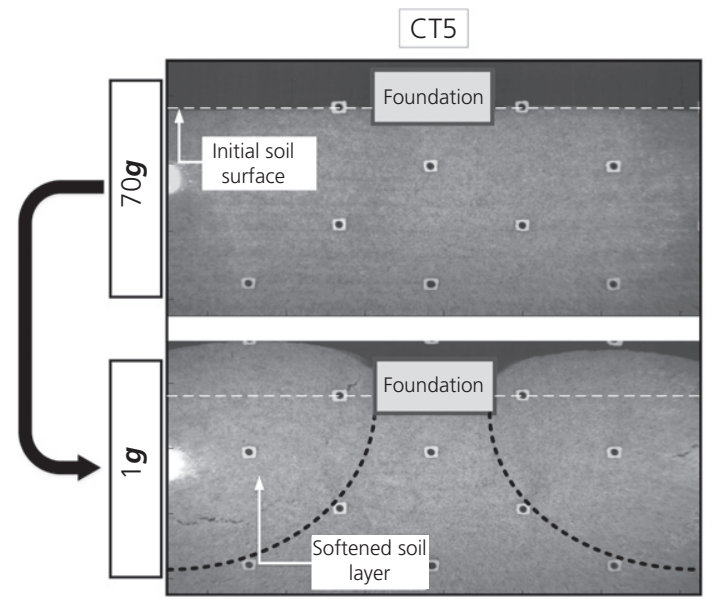

(a)

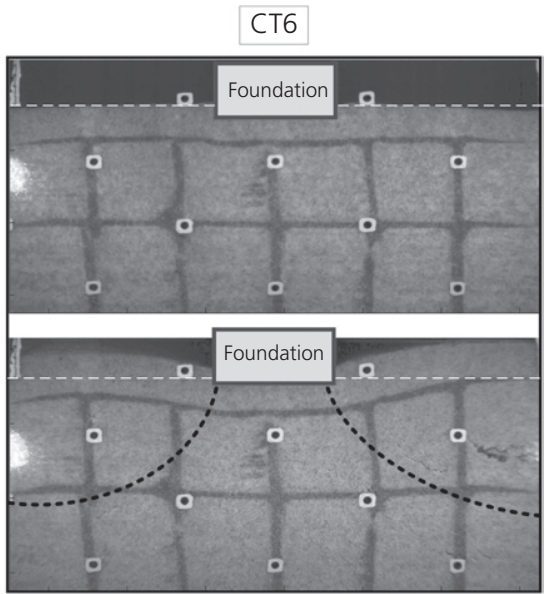

(b)

Figure 9. Images taken at $70 \boldsymbol{g}$ and $1 \boldsymbol{g}$ during centrifuge swings-down 


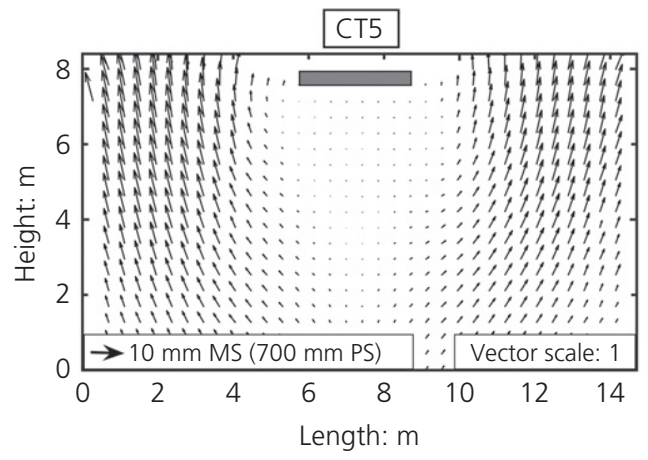

Horizontal displacement: $\mathrm{mm} \quad$ Vertical displacement: $\mathrm{mm}$

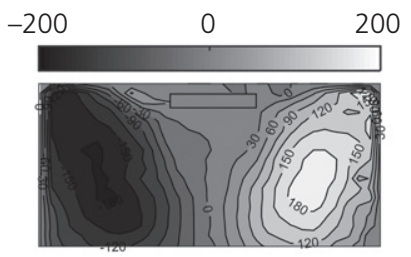

(a)

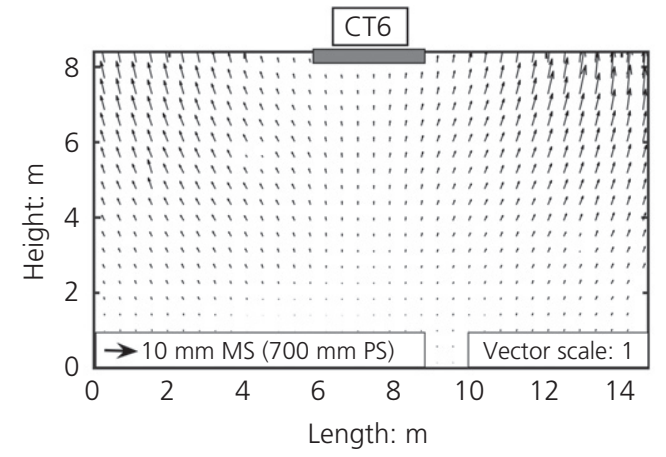

Horizontal displacement: $\mathrm{mm} \quad$ Vertical displacement: $\mathrm{mm}$

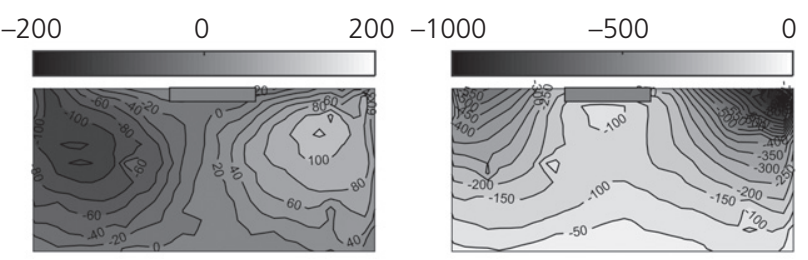

(b)

Figure 10. The displacement vectors accumulated during centrifuge swings-down (prototype scale)

particularly at the shallow layers. Figure 10 presents the horizontal and vertical displacements of the soil particles that took place during this process. It is obvious that the soil particles moved laterally and vertically upwards (negative vertical displacements refer to the soil heaving). The movement of soil particles under the shallow foundations was very small due to the bearing pressure of the foundations. However, the soil particles further away from the edges of the foundations were pushed vertically upwards.

\subsection{Durability under horizontal excitation}

The durability of entrapped air bubbles was investigated under earthquakes. The acceleration-time histories and variation of $S_{\mathrm{r}}$ during the earthquakes in centrifuge tests CT7 and CT8 are illustrated in Figure 11. The variation of $S_{\mathrm{r}}$ was evaluated based on the readings of soil moisture sensors (Vegetronix) placed at the mid-depth of soil layers. By vibrating the partially saturated specimens, it was found that $S_{\mathrm{r}}$ increased by $0.64 \%$ in CT8. In a smaller earthquake, this change was much smaller $(0 \cdot 41 \%)$ in CT7. The increase in $S_{\mathrm{r}}$ during shaking can be mainly ascribed to the compression of the air bubbles during the seismic event due to their highly compressible nature, as indicated by Hsu and Vucetic (2004). Another source of this might be pore fluid flow. It was reported by Zeybek and Madabhushi (2017a) that excess pore pressure gradients generated by earthquakes led to pore fluid flow during and after shaking. The pore fluid flow continued until the pore pressures reached equilibrium in the soil. It can be intuitively suggested that the pore fluid flow might lead to the transport and escape of air bubbles, particularly from the upper part of the soil layers. However, this mechanism is expected to be of secondary importance considering the findings presented in Section 4.2.

\subsection{Settlement of soil and foundation}

The settlement of the free-field and shallow foundation was examined under the combined effects of the aforementioned simulated field conditions in centrifuge test CT9. Two columns of saturated soil with the same $D_{\mathrm{r}}$ were prepared within the same model. They were separated by an impermeable and flexible membrane at the centre of the laminar box. One side of it (section 1) involved the study of partially saturated soil (PS) created through the injection of air, whereas nearly fully saturated soil (FS) was tested in another side (section 3), as shown in Figure 3. The settlements were measured through linear variable differential transformers. This $70 \mathrm{~g}$ centrifuge experiment consisted of two flights. The first flight aimed at testing the free-field settlement of soil deposits under various simulated field conditions that consisted of hydrostatic, fluid pressure increase and earthquake. The second flight involved the investigation of the seismic response of the foundation on the same soil deposits that had been additionally exposed to fluid pressure decrease. For this purpose, a shallow-foundation model with a bearing pressure, $q$, of $135 \mathrm{kPa}$ (prototype scale) was placed on the soil deposit, prior to the second flight. Centrifuge models were subjected to three earthquakes. A peak base acceleration of around $0 \cdot 18 \mathrm{~g}$ (prototype scale) was applied in each case. Figure 12 presents the settlement-time histories obtained at different stages of the experiment. Air was injected once the centrifugal acceleration of $70 \mathrm{~g}$ was reached. The maximum air-injection pressure applied was equal to the sum of the hydrostatic pressure and approximately 

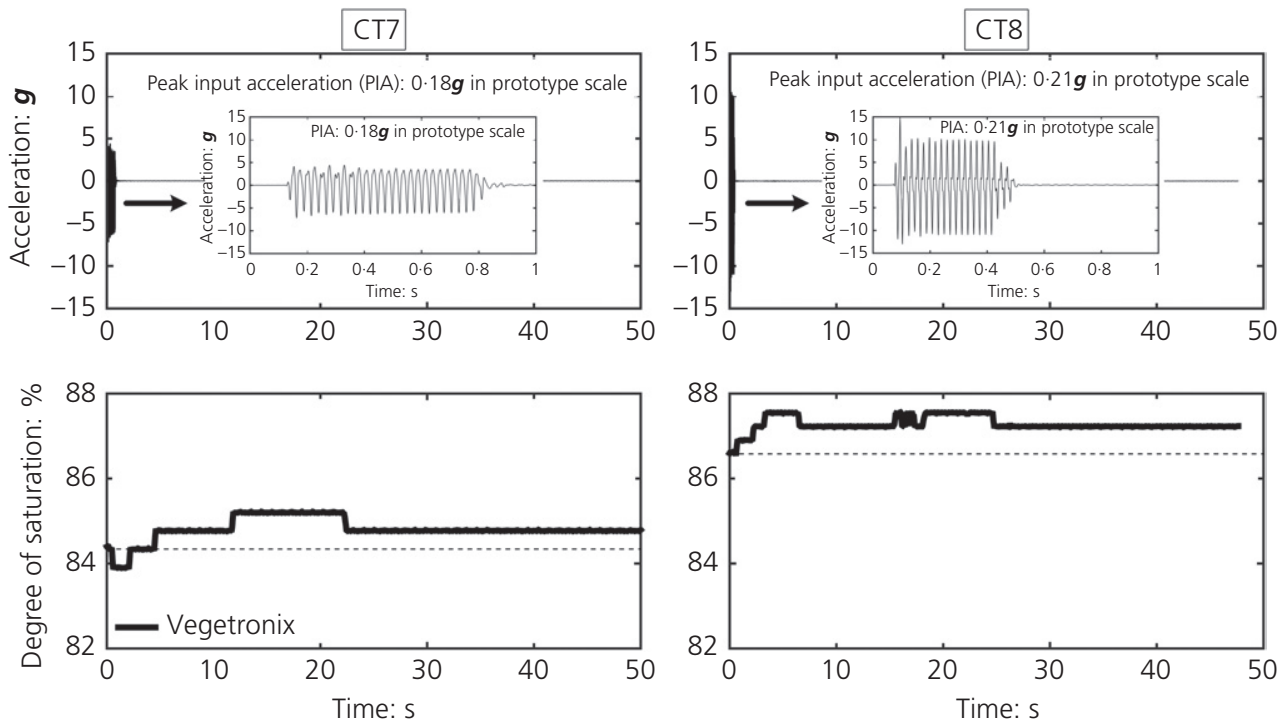

Figure 11. The variation of degree of saturation under horizontal shaking (model scale)
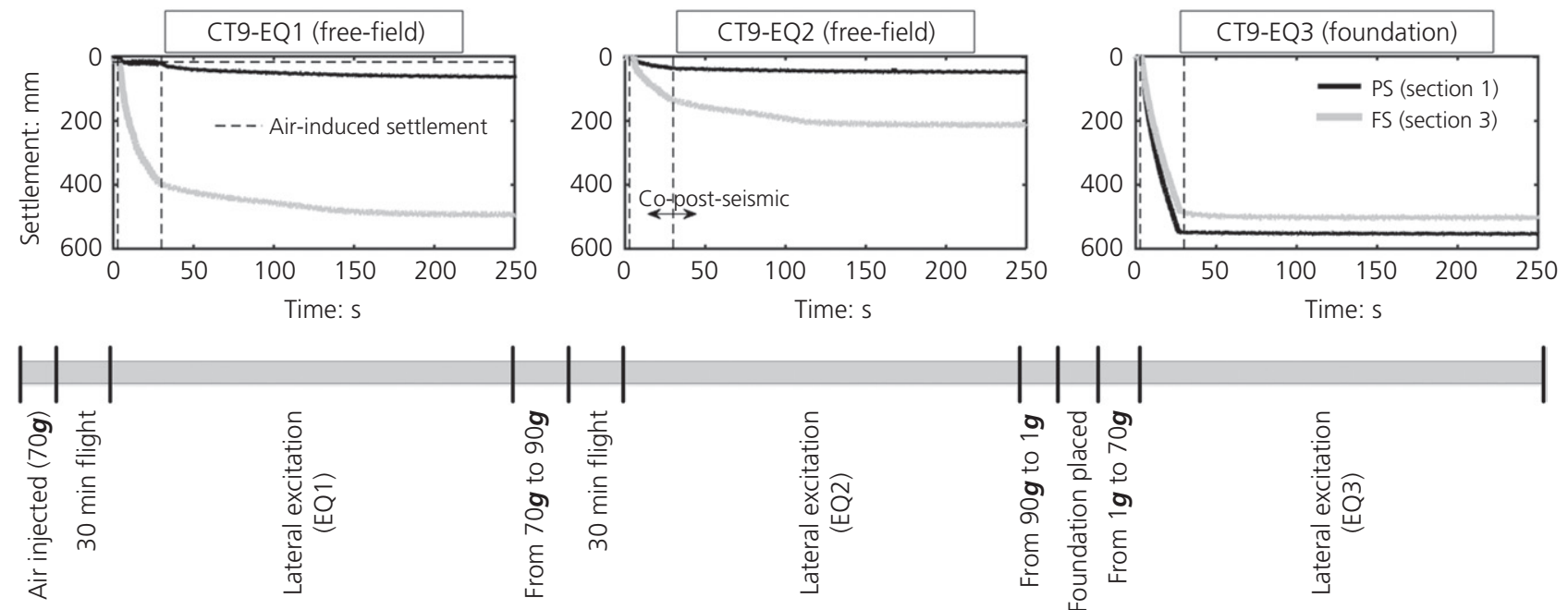

Figure 12. The seismic settlements of free-field and foundation recorded at different stages (prototype scale)

$0 \cdot 23$ times the vertical effective stress at the injection point. This resulted in a free-field settlement of about $15.3 \mathrm{~mm}$, in prototype scale. The air-induced settlement is indicated by a horizontal dashed line in Figure 12. This settlement was attributed to the drop in effective stresses and flow-induced liquefaction in the upper part of soil layers as well as increased compressibility of the soil matrix with air injection (Zeybek and Madabhushi, 2017b). Following the air-injection process, the $S_{\mathrm{r}}$ of soil reduced by $14 \%$ (from 99 to $85 \%$ ). The first earthquake was fired after $30 \mathrm{~min}$ (model scale) of centrifugal flight. It is obvious that the rate of co-seismic and post-seismic free-field settlement of the partially saturated soil was much smaller than that of the nearly fully saturated soil. Moreover, the partially saturated soil experienced a significantly smaller magnitude of total free-field settlement. The second earthquake on the same model was applied after increasing centrifugal acceleration from $70 \mathrm{~g}$ to $90 \mathrm{~g}$ and $30 \mathrm{~min}$ (model scale) of centrifugal flight at $90 \mathrm{~g}$. Similarly, the partially saturated free-field soil settled less than its nearly fully saturated counterpart. These findings may indicate that air injection was still very effective at reducing the liquefaction-induced settlement although the soil layer was exposed to different simulated field conditions. Following the second earthquake, the model was swung down to $1 \mathrm{~g}$. The foundation model was positioned directly on the soil layer and the model was swung up to $70 \mathrm{~g}$ again. The third shaking was applied. The foundation settled more in the partially saturated soil than in the nearly fully saturated soil, which differed from the free-field settlement 
response. Moreover, this was not consistent with the experimental findings of researchers indicated in Section 1. Although these results appear to be out of trend, they highlight the importance of the equilibrium between entrapped air bubbles and the surrounding pore fluid. As reported in Section 4.3, the soil deposit, particularly at the shallow layer, softened due to soil disturbance, and a large foundation settlement was an inevitable corollary of this.

\section{Conclusions}

Although induced partial saturation techniques might offer an economical solution for liquefaction problems, their use in practice is not yet prevalent. This is most notably due to engineering concerns over the long-term durability of gas/air bubbles in soils. The aim of this study was to investigate the possible effects of various field conditions on the durability of entrapped air bubbles. Of particular interest was to examine the seismic performance of shallow foundations resting upon partially saturated soils that were exposed to a combination of several field conditions. For this purpose, a series of $1 g$ vertical sand column and high- $g$ centrifuge tests was performed.

The field scenarios were simplified and simulated in the laboratory. With the simulated field conditions, the durability of air bubbles in specimens was examined under hydrostatic conditions at low and high fluid pressure, upward and downward vertical flow, varying pore fluid pressure and lateral excitation. Analysis of the experimental data suggested that some of the entrapped air bubbles in the partially saturated soils lost their function under these conditions, and this led to an increase in the degree of saturation of the specimens. However, the magnitude of this was generally very small indeed. This was the case for almost all conditions, except one condition under which decreasing $\boldsymbol{g}$-level and therefore pore fluid pressure caused unstable air bubbles and upwards movement of soil particles, leading to significant volume change and deformations in soil deposits.

In the second phase of the research, the seismic settlement of the free-field and shallow foundation was investigated under the combined effects of various simulated field conditions. The analysed data revealed that, although the soil deposit previously improved through air injection was exposed to the majority of the aforementioned field scenarios, air injection was still very effective in reducing the liquefaction-induced free-field settlements. On the other hand, a markedly large settlement of the shallow foundation was observed when it was tested on the same air-injected soil deposit, but had undergone swings-down (decreasing $g$-level). This result highlight the importance of the state of equilibrium between entrapped air bubbles and the surrounding pore fluid. The movement of air bubbles in soils would be possible if the soil or water pressure was reduced significantly.

Although only a simplified version of real field conditions is considered in this paper, it offers valuable hints and insights into the long-term reliability of induced partial saturation techniques. These insights might be of interest to practising engineers working on liquefaction remediation techniques, who may begin the frequent use of these techniques in real engineering projects worldwide.

\section{Acknowledgement}

The centrifuge experiments were performed with the assistance of the technicians at the Schofield Centre of Cambridge University. This support is gratefully acknowledged.

\section{REFERENCES}

Chaney R (1978) Saturation effects on the cyclic strength of sands. In Proceedings of the Earthquake Engineering and Soil Dynamics, ASCE, Pasadena, California, vol. 1, pp. 342-358.

Eseller-Bayat E, Yegian MK, Alshawabkeh A and Gokyer S (2013) Liquefaction response of partially saturated sands. I: experimental results. Journal of Geotechnical and Geoenvironmental Engineering ASCE 139(6): 863-871.

Fredlund DG and Rahardjo H (1993) Soil Mechanics for Unsaturated Soils. John Wiley \& Sons, New York, NY, USA.

Gokyer S (2009) Inducing and Imaging Partial Degree of Saturation in Laboratory Sand Specimens. MS thesis, Northeastern University, Boston, MA, USA.

He J, Chue J and Ivanov V (2013) Mitigation of liquefaction of saturated sand using biogas. Géotechnique 63(4): 267-275, https://doi.org/ 10.1680/geot.SIP13.P.004.

Hsu CC and Vucetic M (2004) Volumetric threshold shear strain for cyclic settlement. Journal of Geotechnical and Geoenvironmental Engineering ASCE 130(1): 58-70.

Madabhushi SPG (2014) Centrifuge Modelling for Civil Engineers. Taylor \& Francis, London, UK.

Madabhushi SPG, Schofield AN and Lesley S (1998) A new stored angular momentum (SAM) based earthquake actuator. In Proceedings of Centrifuge ' 98 (Kimura T, Kusakabe $\mathrm{O}$ and Takemura J (eds)). Balkema, Tokyo, Japan, vol. 1, pp. 111-116.

Okamura M and Soga Y (2006) Effects of pore fluid compressibility on liquefaction resistance of partially saturated sand. Soils and Foundations 46(5): 695-700.

Okamura M and Tomida Y (2015) Full scale test on cost effective liquefaction countermeasure for highway embankment. In Proceedings of the 6th International Geotechnical Symposium on Disaster Mitigation in Special Geoenvironmental Conditions, IIT Madras, Chennai, India (published by Indian Geotechnical Society, Chennai Chapter), vol. 1, pp. 208-212.

Okamura M, Ishihara M and Tamura K (2006) Degree of saturation and liquefaction resistances of sand improved with sand compaction pile. Journal of Geotechnical and Geoenvironmental Engineering ASCE 132(2): 258-264.

Okamura M, Takebayashi M, Nishida K et al. (2011) In-situ desaturation test by air injection and its evaluation through field monitoring and multiphase flow simulation. Journal of Geotechnical and Geoenvironmental Engineering ASCE 137(7): 643-652.

White DJ, Take WA and Bolton MD (2003) Soil deformation measurement using particle image velocimetry (PIV) and photogrammetry. Géotechnique 53(7): 619-631, https://doi.org/ 10.1680/geot.2003.53.7.619.

Yegian MK, Eseller-Bayat E, Alshawabkeh A and Ali S (2007) Induced-partial saturation for liquefaction mitigation: experimental investigation. Journal of Geotechnical and Geoenvironmental Engineering ASCE 133(4): 372-380. 
Zeybek A and Madabhushi SPG (2017a) Centrifuge testing to evaluate the liquefaction response of air-injected partially saturated soils beneath shallow foundations. Bulletin of Earthquake Engineering 15(1): 339-356.
Zeybek A and Madabhushi SPG (2017b) Influence of air injection on the liquefaction-induced deformation mechanisms beneath shallow foundations. Soil Dynamics and Earthquake Engineering, http://dx.doi.org/10.1016/j.soildyn.2017.03.018.

\section{How can you contribute?}

To discuss this paper, please email up to 500 words to the editor at journals@ice.org.uk. Your contribution will be forwarded to the author(s) for a reply and, if considered appropriate by the editorial board, it will be published as discussion in a future issue of the journal.

Proceedings journals rely entirely on contributions from the civil engineering profession (and allied disciplines). Information about how to submit your paper online is available at www.icevirtuallibrary.com/page/authors, where you will also find detailed author guidelines. 